\title{
Understanding asthma-specific quality of life: moving beyond asthma symptoms and severity
}

\author{
Brian D. Stucky ${ }^{1}$, Cathy D. Sherbourne ${ }^{1}$, Maria Orlando Edelen ${ }^{2}$ and \\ Nicole K. Eberhart ${ }^{1}$
}

Affiliations:

${ }^{1}$ RAND Corporation, Santa Monica, CA, USA.

${ }^{2}$ RAND Corporation, Boston, MA, USA.

\section{Correspondence:}

Brian Stucky, RAND Corporation, 1776 Main Street, PO Box 2138, Santa Monica, CA, 90407, USA.

E-mail: bstuckyarand.org

ABSTRACT This study identifies the unique contributions of asthma severity, symptoms, control and generic measures of quality of life (QoL) to asthma-specific QoL, as measured by the 12-item RAND Negative Impact of Asthma on Quality of Life scale (RAND-IAQL-12).

Using a sample of 2032 adults with asthma, we conducted multiple regression analyses that sequentially examined hypothesised predictors of asthma-specific QoL. The change in variance accounted for and total unique variance accounted for is calculated as hypothesised predictors are added in each step.

Our results indicate that asthma severity and asthma symptoms are strong predictors of asthma-specific QoL only when not controlling for aspects of asthma control. In regression models that include other aspects of asthma control, the contributions of both asthma symptoms and severity were substantially reduced, with asthma control and aspects of QoL related to social roles and activities emerging as the strongest predictors of asthma-specific QoL.

These findings suggest that researchers measuring the impact of asthma on QoL should also consider the importance of asthma control as measured by the RAND Asthma Control Measure (RAND-ACM) and generic QoL scales that measure aspects of daily life that are uniquely affected by asthma.

@ERSpublications

It is the impact of asthma symptoms on daily life, rather than symptoms themselves, that most affect quality of life http://ow.ly/KGIO4 


\section{Introduction}

Asthma has been shown to impair a person's quality of life (QoL), and the burden of asthma on QoL may provide complementary information along with more commonly used measures of asthma symptoms, severity and control [1]. An expert panel convened by the National Institutes of Health (NIH) in 2010 stated that "because the burden of asthma as measured by symptom or activity levels does not give a complete picture, an assessment of the patient's perception of the impact of impairments on his or her QoL remains essential [2]." The Asthma Quality of Life Subcommittee of the $2010 \mathrm{NIH}$ Asthma Outcomes Workshop suggested that identifying the aspects of asthma control (e.g. symptom intensity and frequency, impairment in physical activities, etc.) that predict asthma-specific QoL may provide important information for clinicians and researchers.

Many prior studies have evaluated the relationships of asthma-specific QoL with measures of asthma control [3-9]. For example, several studies have found that indicators of asthma control are highly correlated with asthma-specific QoL $(\mathrm{r}=0.49-0.78)[3,5,8]$ and may explain $54-58 \%$ of the variance in asthma-specific QoL [9]. However, the majority of this research used measures of asthma-specific QoL that contained aspects of asthma control (e.g. activity limitations and asthma symptoms [10]); a shortcoming highlighted by the $2010 \mathrm{NIH}$ workshop [2]. For example, CHEN et al. [3] report that as much as $44 \%$ of the variability in asthma-specific QoL could be accounted for by demographic and comorbid conditions, asthma control, severity and preference-based generic QoL; however, the outcome measure used was the Mini Asthma Quality of Life Questionnaire (mini-AQLQ) [11], which contains both asthma symptoms and activity limitations subdomains. This overlap with indicators of asthma control may have inflated the association between control and asthma-specific QoL. Thus, the degree to which the strong associations between asthma control and asthma-specific QoL are simply an artefact of the traditional measures that contain overlapping content remains unclear.

To address this concern and in response to the $2010 \mathrm{NIH}$ workshop recommendations regarding confounding asthma symptoms with QoL, we developed a new measure of asthma-specific QoL (The RAND Negative Impact of Asthma on Quality of Life (RAND-IAQL)) that assesses the degree of asthma impact or burden on QoL [12-14]. Developed using item-response theory, the new system consists of a 65-item item-bank and 4- and 12-item short forms. Preliminary evidence indicates that the RAND-IAQL differentiates between adults with asthma according to various comorbid conditions and perceived severity [13]. Unlike traditional measures of asthma-specific QoL the items in the RAND-IAQL were developed to avoid confounding QoL with asthma symptomatology and functional impairment, while focussing on content areas that reflect domains of life important to people with asthma [12,13]. Because the RAND-IAQL was developed to avoid confounding QoL with asthma symptomology and severity, it serves as an unbiased outcome when used to differentially predict asthma-specific QoL from aspects of asthma control, including functional impairment, symptoms and severity. However, to date, no studies have systematically evaluated the impact of asthma control on asthma-specific QoL as measured by the RAND-IAQL.

One of the challenges in evaluating these relationships is that there is considerable overlap in the definitions of asthma symptoms, severity and control [15-19]. Asthma symptoms are traditionally assessed based on the frequency of wheezing, coughing, chest discomfort, etc. Recent guidelines define well-controlled asthma based on a lack of asthma symptoms, as well as the minimal need for use of a rescue inhaler, no night-time awakenings, normal lifestyle and minimal airway obstruction [20]. Because of the overlap in these definitions, it is common practice for researchers to treat asthma control as a composite measure consisting of aspects of asthma symptoms, treatment requirements, severity, and impact on daily functioning [21]. However, this approach ignores that certain components of asthma control (e.g. functional limitations) may be more predictive of asthma-specific QoL than other components of asthma control (e.g. asthma symptoms). Thus, a separate goal of the present study is to identify the particular aspects of asthma control, symptoms and severity that predict asthma-specific QoL.

In this paper, we use regression models to evaluate the degree to which asthma-specific QoL (as measured by the RAND-IAQL-12) is predicted by generic measures of QoL (i.e. non-disease specific QoL), asthma symptoms, asthma control, comorbid conditions commonly associated with asthma, emergency room visits and self-rated asthma severity. The primary goal was to evaluate the relationship between asthma control and asthma-specific QoL that is not confounded by asthma symptoms. A separate goal was to understand the degree to which asthma symptoms are uniquely related to asthma-specific QoL above and beyond the composite measure of asthma control. While many studies have evaluated the independent or marginal associations between control and asthma-specific QoL, fewer studies have evaluated the degree to which the asthma symptom component of asthma control is related to asthma-specific QoL. For example, because many of these asthma-related factors are intercorrelated, it is possible that their seemingly strong associations with asthma-specific QoL are actually much smaller in magnitude when taking into account a range of asthma-related covariates. 


\section{Materials and methods Study subjects}

A national sample of adults (aged $\geqslant 18$ years) with asthma $(\mathrm{n}=2032)$ completed an internet-based survey assessment. Participant eligibility was satisfied if: 1) they had been told by a doctor/health professional that they had asthma; and 2) they reported still having asthma. To ensure variability in asthma severity levels, we required that $90 \%$ of the sample had experienced an episode of asthma or an asthma attack during the past year [22]. In addition to oversampling minorities, we achieved a sample of about $40 \%$ men to reflect the distribution of individuals with asthma in the general adult population in the USA. In order to avoid potential confounds with chronic obstructive pulmonary disease (COPD) with asthma, we limited the proportion of the sample that had comorbid COPD. To incentivise study participation, participants received points through the online survey vendor that could then be redeemed for rewards.

Table 1 describes the participants' demographic characteristics, medical comorbidities, emergency room visits, indicators of asthma severity, symptoms, control and medication use, along with the QoL scores. In the past year, $39 \%(n=785)$ of the sample had visited an emergency room or urgent care facility. A majority of the sample reported having at least one chronic medical condition in addition to their asthma, with $28 \%(n=570)$ reporting having two medical comorbidities, and $n=297(15 \%)$ having more than two medical comorbidities.

\section{Measures}

Asthma severity: emergency room visits and self-report severity

As proxy variables for asthma severity, participants were asked if they visited an emergency room in the past year because of an asthma event or attack. An additional item asked participants to rate their asthma severity (five-point response scale with higher scores indicating more severe asthma; table 1).

\section{Asthma symptoms}

Asthma symptoms were assessed with a composite of two items assessing the frequency of wheezing or coughing due to asthma (Cronbach's $\alpha=0.72$ ). Both items use five-point Likert scales where higher scores indicate more frequent symptoms.

\section{Asthma control}

Asthma control was assessed using the five-item RAND Asthma Control Measure (RAND-ACM) [23] that was developed in light of recent guidelines by the Global Initiative for Asthma (GINA) [24] and the US National Heart, Lung, and Blood Institute (NHLBI) [25]. The RAND-ACM items measure frequency of asthma attacks, being awakened at night because of asthma, asthma interference on normal activities, use of rescue inhalers and shortness of breath. Scores for the RAND-ACM have demonstrated adequate reliability (Cronbach's $\alpha=0.84$ ) and closely fit a unidimensional model [23]. The items are on four- and five-point Likert type scales with higher scores indicating less asthma control. Using NHLBI guidelines, according to the RAND-ACM, $21 \%$ of the current sample had well-controlled asthma, $44 \%$ had poorly controlled asthma and $35 \%$ had very poorly controlled asthma (table 1 ).

\section{Quality of life}

Three measures of QoL were assessed. Participants' perception of their general QoL were measured with a single item (i.e. "In general, would you say your quality of life is...?"; higher scores mean better QoL; table 1). Two additional QoL scales were included to assess aspects of generic QoL that may be impacted by asthma: the Patient Reported Outcomes Measurement Information System (PROMIS) Anxiety [26] and PROMIS Ability to Participate in Social Roles/Activities [27] four-item short-forms (Cronbach's $\alpha=0.94$ and 0.95 , respectively; available at www.assessmentcenter.net). The Anxiety short form includes feeling fearful, worried, etc. The Social Roles/Activities short form measures difficulties doing activities with others (e.g. "I have trouble doing all of the activities with friends that I want to do"). Both scales are scored on a standardised T-score metric (calibrated to have a mean of 50 and standard deviation of 10). For the Social Roles/Activities short form, higher scores indicate better QoL, and for the Anxiety short form higher scores indicate lower QoL.

\section{Asthma-specific QoL}

The RAND-IAQL measurement system consists of an item bank (i.e. a large set of items with known characteristics that measure the same underlying construct), a brief four-item short form that assesses global aspects of asthma-specific QoL (RAND-IAQL-4), and a 12-item short form with broader content coverage that provides more measurement precision. The RAND-IAQL system was developed using extensive qualitative, including focus groups, cognitive testing, expert review and literature review [12], and quantitative methods including factor analysis to establish unidimensionality, and item-response theory and 
TABLE 1 Demographic characteristics of asthma sample

\begin{tabular}{|c|c|}
\hline Participants n & 2032 \\
\hline Age & $43 \pm 15$ \\
\hline \multicolumn{2}{|l|}{ Sex } \\
\hline Female & $1219(60)$ \\
\hline \multicolumn{2}{|l|}{ Ethnicity/race } \\
\hline Hispanic & $282(14)$ \\
\hline Asian & $221(11)$ \\
\hline African-American & 381 (19) \\
\hline Non-Hispanic white & $1148(56)$ \\
\hline \multicolumn{2}{|l|}{ Region of USA } \\
\hline East & $478(24)$ \\
\hline Midwest & $432(21)$ \\
\hline South & $578(28)$ \\
\hline West & 544 (27) \\
\hline \multicolumn{2}{|l|}{ Education } \\
\hline Up to high school & 353 (17) \\
\hline Some college & $743(36)$ \\
\hline College graduate & $490(24)$ \\
\hline Some graduate school or graduate degree & $446(22)$ \\
\hline \multicolumn{2}{|l|}{ Medical comorbidities } \\
\hline Sinusitis & $1284(63)$ \\
\hline Diabetes & 505 (25) \\
\hline Heart disease & 194 (9) \\
\hline COPD & $287(14)$ \\
\hline Migraine headaches & 660 (32) \\
\hline Exactly one condition & 745 (37) \\
\hline Exactly two conditions & $570(28)$ \\
\hline$\geqslant$ Three conditions & $297(15)$ \\
\hline Visited emergency room in past year & 783 (39) \\
\hline \multicolumn{2}{|l|}{ Self-rating of asthma severity } \\
\hline Very mild & 383 (19) \\
\hline Mild & $580(28)$ \\
\hline Moderate & $824(41)$ \\
\hline Severe & $207(10)$ \\
\hline Very severe & $36(2)$ \\
\hline \multicolumn{2}{|l|}{ Rescue inhaler use during past 4 weeks } \\
\hline Never & $495(24)$ \\
\hline 1 time per week & $510(25)$ \\
\hline 2 or more times per week but not daily & $600(29)$ \\
\hline Daily & 279 (14) \\
\hline Several times per day most days & $143(7)$ \\
\hline \multicolumn{2}{|l|}{ RAND-ACM (using NHLBI guidelines) } \\
\hline Well-controlled (5-7) & $429(21)$ \\
\hline Not well-controlled (8-12) & $879(44)$ \\
\hline Very poorly controlled (13-24) & 702 (35) \\
\hline \multicolumn{2}{|l|}{ General quality of life } \\
\hline Excellent & $109(5)$ \\
\hline Very good & 370 (18) \\
\hline Good & 770 (38) \\
\hline Fair & 591 (29) \\
\hline Poor & $185(9)$ \\
\hline \multicolumn{2}{|l|}{ Specific quality of life } \\
\hline Social roles/activities (T-scale) ${ }^{\#}$ & $48.8 \pm 9.0$ \\
\hline Anxiety $(T-\text { scale })^{\#}$ & $54.2 \pm 11.6$ \\
\hline
\end{tabular}

Data are presented as $\mathrm{n}(\%)$ or mean $\pm \mathrm{SD}$, unless otherwise stated. The mean item response for asthma symptoms and asthma control on a 0-4 scale is reported here. COPD: chronic obstructive pulmonary disease; RAND-ACM: RAND Asthma Control Measure; NHLBI: National Heart, Lung, and Blood Instritute. \#: The Patient Reported Outcomes Measurement Information System Social Roles/Activities and Anxiety measures were scored using a standardised T-score metric with a mean of 50 and standard deviation of 10. 
simulated computer adaptive testing [14]. Preliminary evidence suggests that the RAND-IAQL is highly correlated with legacy measures of asthma-specific QoL and discriminates between adults according level of severity and control status [13]. The current study makes use of the RAND-IAQL-12, which includes items related to physical limitations, social concerns, inhaler awareness, health concerns, sleep difficulties and three global items (marginal reliability=0.93) [14]. The items have a consistent timeframe (past 4 weeks), orientation (first-person), and response format (five-point Likert-type) reflecting magnitude (i.e. "not at all" to "very much") or frequency of impact (i.e. "never" to "almost always"). Because the RAND-IAQL-12 measures the negative impact of asthma on QoL, higher scores indicate more impact of asthma on QoL, thus lower scores on the RAND-IAQL-12 indicate higher asthma-specific QoL (for additional details and to obtain the complete set of measures, visit www.rand.org/health/surveys_tools/iaql.html).

\section{Missing data}

Though there was little missing data (all items had $<3 \%$ missing), multiple imputation methods were used to maintain a consistent sample size across regression models. Based on the assumption that item responses were missing at random, the imputation model (based on MCMC to draw imputed values as implemented in the SAS procedure MI) included all independent variables and generated 20 imputed datasets.

Analysis

A series of linear regression models was estimated and then synthesised using standard methods in the SAS procedure MIANALYZE. Each model builds on the prior model by adding a component that is hypothesised to predict asthma-specific QoL. The goal of the modelling approach was to show the patterns of change in the regression coefficients as additional variables are included in the models, thus highlighting the unique influence of each component. Demographic variables and comorbid conditions were added first to serve as covariates for later models (i.e. Model 1). Model 2 adds the proxy measures of asthma severity, which should be taken into account prior to assessing asthma control. Asthma control was next added to the model (Model 3) to evaluate their relationship with asthma-specific QoL when not accounting specifically for asthma symptoms or generic aspects of QoL. We then added asthma symptoms (Model 4) to evaluate the degree to which they are uniquely related to asthma-specific QoL above and beyond the effect of other aspects of asthma control, severity and the other covariates. Finally, Model 5 adds three measures of generic QoL: 1) general QoL, which is expected to account for variance in asthma-specific QoL that is operationally different from the asthma-specific variables already included the model, 2) social activity limitations, which is included to account for the impact of asthma on social relationships and obligations, and 3) anxiety which we hypothesised would be related to the worry associated with managing asthma. Typical regression assumptions were tested for violations using Model 5 (e.g. normality, linearity, multicollinearity and homoscedasticity).

\section{Results}

Results are displayed for the individual standardised regression coefficients which reflect the change in strength between the components in the model as additional predictors are added, and also for the total and unique variance accounted for in asthma-specific QoL that is sequentially contributed by each model. The statistical significance of the difference in variance accounted for by each addition to the model is evaluated by partial F-tests.

\section{Model 1}

The regression results for the baseline model (Model 1; table 2) show that demographic variables and comorbid conditions account for $20 \%$ of the variance in the RAND-IAQL-12. Ethnicity and sex were reference-cell coded with minority ethnicity and males serving as the reference groups. Results indicated that females and non-Hispanic white subjects were more likely to have lower impact of asthma on QoL ( $i$. $e$. more positive outcomes) than males and minority ethnic groups after accounting for all other variables in the model (0.09 SDS and 0.24 SDs lower, respectively). In addition, older individuals and those with higher education were also associated with lower impact on asthma QoL scores (i.e. positive outcomes). Increased number of medical comorbidities was related to significantly greater impact asthma QoL scores (i.e. negative outcomes) (table 2).

\section{Model 2}

The inclusion of self-perceived severity and emergency room visits (proxies for asthma severity) results in a statistically significant $32 \%$ increase in the variance accounted for by the model (table 3 ). Self-perceived severity and having visited an emergency room in the past year were both associated with significantly greater impact of asthma on QoL (table 2) and resulted in a model accounting for $52 \%$ of the variance in the impact of asthma on QoL. 
TABLE 2 Regression coefficients depicting the relationship between each component in the model and the negative impact of asthma on quality of life

$\begin{array}{ccccc}\text { Model } 1 & \text { Model 2 } & \text { Model 3 } & \text { Model 4 } & \text { Model 5 } \\ \left(R^{2}=0.20\right) & \left(R^{2}=0.52\right) & \left(R^{2}=0.65\right) & \left(R^{2}=0.65\right) & \left(R^{2}=0.75\right)\end{array}$

\begin{tabular}{|c|c|c|c|c|c|}
\hline \multicolumn{6}{|l|}{ Model 1: demographic and comorbid conditions } \\
\hline Ethnic minority status ${ }^{\#}$ & $-0.24 \pm 0.02$ & $-0.09 \pm 0.02$ & $-0.08 \pm 0.01$ & $-0.08 \pm 0.01$ & $-0.05 \pm 0.01$ \\
\hline Sex & $-0.09 \pm 0.02$ & $-0.06 \pm 0.02$ & $-0.05 \pm 0.01$ & $-0.05 \pm 0.01$ & $-0.06 \pm 0.01$ \\
\hline Age & $-0.19 \pm 0.02$ & $-0.07 \pm 0.02$ & $-0.09 \pm 0.01$ & $-0.09 \pm 0.01$ & $-0.08 \pm 0.01$ \\
\hline Comorbid conditions & $0.25 \pm 0.02$ & $0.11 \pm 0.02$ & $0.06 \pm 0.01$ & $0.06 \pm 0.01$ & $-0.01 \pm 0.01$ \\
\hline \multicolumn{6}{|l|}{ Model 2: asthma severity } \\
\hline Self-perceived asthma severity & & $0.46 \pm 0.02$ & $0.18 \pm 0.02$ & $0.18 \pm 0.02$ & $0.14 \pm 0.02$ \\
\hline Model 4: asthma symptoms & & & & $-0.00 \pm 0.02$ & $0.00 \pm 0.02$ \\
\hline \multicolumn{6}{|l|}{ Model 5: PROMIS overall health and quality of life scales } \\
\hline PROMIS General Quality of Life ${ }^{+}$ & & & & & $-0.02 \pm 0.01$ \\
\hline PROMIS Ability to Participate in Social Roles/Activities ${ }^{+}$ & & & & & $-0.32 \pm 0.02$ \\
\hline PROMIS Anxiety & & & & & $0.15 \pm 0.02$ \\
\hline
\end{tabular}

\section{Model 3}

The addition of asthma control marks a statistically significant increase in the variance accounted for by the model (an additional 13\% unique variance and 65\% total variance; tables 2 and 3). At this point, asthma control is noted as the strongest predictor of asthma-specific QoL.

\section{Model 4}

The inclusion of asthma symptoms did not result in a statistically significant increase in the variance accounted for by the model (table 3).

\section{Model 5}

Among the generic QoL variables included in Model 5, the ability to participate in social roles is clearly the greatest contributor to the prediction of asthma-specific QoL, while anxiety has a modest relationship and general QoL only weakly contributes to the variance accounted for in the RAND-IAQL-12. The final model accounts for $75 \%$ of the variance in asthma-specific QoL (table 2).

\section{Model review}

Table 3 displays both the sequential increase in the variance of RAND-IAQL-12 accounted for by each added step in the model, and the unique variance of RAND-IAQL-12 accounted for by each aspect of the

\section{TABLE 3 The unique contribution of variables predicting the negative impact of asthma on quality of life}

\begin{tabular}{lcc} 
Evaluated model & $\Delta \mathbf{R}^{2}$ & Unique $\mathbf{R}^{2}$ \\
\hline Model 1: Demographics and comorbid conditions & & 0.01 \\
Model 2: Adding Asthma severity & 0.32 & 0.02 \\
Model 3: Adding Asthma control & 0.13 & 0.04 \\
Model 4: Adding Asthma symptoms & 0.00 & 0.00 \\
Model 5: Adding Quality of life & $\mathbf{0 . 1 0}$ & $\mathbf{0 . 1 0}$ \\
\hline
\end{tabular}

Data in bold are statistically significant at $p<0.05$ based on a partial F-tests. $\Delta R^{2}$ : change in $R^{2}$ between the current model and the prior model; unique $R^{2}$ : unique portion of the variance in the negative impact of asthma on quality of life that is contributed by each variable subset in the final model. 
model. The unique variance accounted for by each component in the model is the difference in the amount of variance accounted for by the full model and the amount of variance accounted for when the given component is removed. Arranged in this manner, it is clear that the generic QoL measures account for the most unique variance (10\%) followed by asthma control (4\%). In the full model, very little unique information about asthma-specific QoL is provided by asthma symptoms (0\%) and demographic and comorbid conditions (1\%).

\section{Discussion}

This study examined the differential contributions of asthma severity, symptoms, control and generic QoL to asthma-specific QoL. Our modelling approach indicates that it is the non-symptom components of asthma control and generic QoL (specifically social roles/activities) that account for the greatest proportion of variance in asthma-specific QoL and are therefore critical to the assessment of the impact of asthma on patient well-being.

While our study highlights the importance of measuring asthma control, asthma control is a multidimensional construct with no gold standard of assessment [18]. Because studies often conflate asthma control with asthma severity $[18,28]$, and symptom assessment is a necessary component of asthma control assessment, it is particularly challenging to disentangle the relationship between each individual component and asthma-specific QoL. Importantly, the control measure reported in this study was selected because it is not overly influenced by asthma symptoms. We found that the RAND-ACM along with the PROMIS Ability to Participate in Social Roles/Activities short form were most predictive of asthma-specific QoL. Both of these scales include items measuring the impact of asthma on daily functioning, a finding that is consistent with the asthma control literature. For example, Vollmer et al. [6], Katz et al. [7], SChatz et al. [8] and KING et al. [9] all report that activity limitations explained most variance in traditional measures of asthma-specific QoL (e.g. AQLQ-M [29], AQLQ [30], and mini-AQLQ [11]). However, unlike these prior investigations, the current study is the first to identify the differential relations between perceived asthma severity, symptoms, control, generic aspects of QoL and asthma-specific QoL.

Our study has some notable strengths, including examination of a large and diverse sample of over 2000 individuals, and the use of a new measure of asthma-specific QoL developed using advanced measurement techniques with preliminary evidence of validity. However, it also has some key limitations. First, our models did not include any clinical measures of lung function. However, studies have demonstrated a weak relationship between lung function and asthma-specific QoL $[5,31,32]$, so we believe our models capture the key contributors. Second, because we did not have clinical data about the subject's status prior to treatment or after optimal therapy was established, we were limited to a self-reported measure of severity. It should also be noted that this is a cross-sectional study and, as such, the direction of causality is uncertain. For example, we hypothesise that asthma control influences asthma-specific QoL but, without longitudinal data, this assumption cannot be directly tested. Lastly, it is important to note that our final model accounted for $75 \%$ of the variance in asthma-specific QoL, leaving $25 \%$ of the variance unexplained. This suggests that, while asthma control and generic QoL are key contributors to asthma-specific QoL, asthma-specific QoL is a distinct construct. As such, researchers and clinicians seeking to understand the impact of asthma on individuals should utilise multiple outcome measures, including measures of both asthma control and asthma-specific QoL.

\section{References}

1 Reddel HK, Taylor DR, Bateman ED, et al. An Official American Thoracic Society/European Respiratory Society Statement: Asthma Control and Exacerbations: Standardizing Endpoints for Clinical Asthma Trials and Clinical Practice. Am J Respir Crit Care Med 2009; 180: 59-99.

2 Wilson SR, Rand CS, Cabana MD et al. Asthma outcomes: quality of life. J Allergy Clin Immunol 2012; 129: S88-S123.

3 Chen H, Gould MK, Blanc PD, et al. Asthma control, severity, and quality of life: quantifying the effect of uncontrolled disease. J Allergy Clin Immunol 2007; 120: 396-402.

4 Bateman ED, Bousquet J, Keech ML, et al. The correlation between asthma control and health status: the GOAL study. Eur Respir J 2007; 29: 56-63.

5 Apfelbacher CJ, Jones C, Hankins M, et al. Validity of two common asthma-specific quality of life questionnaires: Juniper mini asthma quality of life questionnaire and Sydney asthma quality of life questionnaire. Health Qual Life Outcomes 2012; 10: 97.

6 Vollmer WM, Markson LE, O'Connor E, et al. Association of asthma control with health care utilization and quality of life. Am J Respir Crit Care Med 1999; 160: 1647-1652.

7 Katz PP, Yelin EH, Eisner MD, et al. Perceived control of asthma and quality of life among adults with asthma. Ann Allergy Asthma Immunol 2002; 89: 251-258.

8 Schatz M, Mosen DM, Kosinski M, et al. The relationship between asthma-specific quality of life and asthma control. J Asthma 2007; 44: 391-395.

9 King MT, Kenny PM, Marks GB. Measures of asthma control and quality of life: longitudinal data provide practical insights into their relative usefulness in different research contexts. Qual Life Res 2009; 18: 301-312. 
10 National Asthma Education and Prevention Program, Third Expert Panel on the Diagnosis and Management of Asthma. Expert Panel Report 3: Guidelines for the Diagnosis and Management of Asthma. Bethesda, National Heart, Lung, and Blood Institute, 2007.

11 Juniper EF, Guyatt GH, Cox FM, et al. Development and validation of the mini asthma quality of life questionnaire. Eur Respir J 1999; 14: 32-38.

12 Eberhart NK, Sherbourne CD, Edelen MO, et al. Development of a measure of asthma-specific quality of life among adults. Qual Life Res 2014; 23: 837-848.

13 Sherbourne CD, Stucky BD, Edelen MO, et al. Assessing the validity of the RAND negative impact of asthma on quality of life short forms. J Allergy Clin Immunol 2014; 134: 900-907.

14 Stucky BD, Edelen MO, Sherbourne CD, et al. Developing an item bank and short forms that assess the impact of asthma on quality of life. Respir Med 2014; 108: 252-263.

15 Cockcroft DW, Swystun VA. Asthma control versus asthma severity. J Allergy Clin Immunol 1996; 98: 1016-1018.

16 Humbert M, Holgate S, Boulet LP, et al. Asthma control or severity: that is the question. Allergy 2007; 62: 95-101.

17 Fuhlbrigge AL. Asthma severity and asthma control: symptoms, pulmonary function, and inflammatory markers. Curr Opin Pulm Med 2004; 10: 1-6.

18 Vollmer WM. Assessment of asthma control and severity. Ann Allergy Asthma Immunol 2004; 93: 409-414.

19 Stoloff SW, Boushey HA. Severity, control, and responsiveness in asthma. J Allergy Clin Immunol 2006; 117: 544-548.

20 Taylor DR, Bateman ED, Boulet LP, et al. A new perspective on concepts of asthma severity and control. Eur Respir J 2008; 32: 545-554.

21 Cloutier MM, Schatz M, Castro M, et al. Asthma outcomes: composite scores of asthma control. J Allergy Clin Immunol 2012; 129: S24-S33.

22 US Centers for Disease Control. America Breathing Easier 2010: CDC's National Asthma Control Program at a Glance. National Center for Environmental Health (NCEH); Division of Environmental Hazards and Health Effects, 2010.

23 Lara M, Edelen MO, Eberhart NK, et al. Development and validation of the RAND Asthma Control Measure (RAND-ACM). Eur Respir J 2014; 44: 1243-1252.

24 Global Initiative for Asthma. Global strategy for asthma managmenent and prevention 2006. www.ginasthma.com Date last accessed: November 2014.

25 National Heart, Lung, and Blood Institute. National Asthma Education and Prevention Program. Expert Panel Report 3: Guidelines for the Diagnosis and Management of Asthma 2007. www.nhlbi.nih.gov/health-pro/ guidelines/current/asthma-guidelines/full-report.htm Date last accessed: November, 2014.

26 Pilkonis PA, Choi SW, Reise SP, et al. Item banks for measuring emotional distress from the Patient-Reported Outcomes Measurement Information System (PROMIS ${ }^{\circledR}$ ): depression, anxiety, and anger. Assessment 2011; 18: 263-283.

27 Bode RK, Hahn EA, DeVellis R, et al. Measuring participation: the patient-reported outcomes measurement information system experience. Arch Phys Med Rehabil 2010; 91: S60-S65.

28 Cockcroft DW, Sywstun VA. Asthma control vs. asthma severity. J Allergy Clin Immunol 1996; 98: 1016-1018.

29 Marks GB, Dunn SM, Woolcock AJ. A scale for the measurement of quality of life in adults with asthma. J Clin Epidemiol 1992; 45: 461-472.

30 Juniper EF, Buist AS, Cox FM, et al. Validation of a standardized version of the Asthma Quality of Life Questionnaire. Chest 1999; 115: 1265-1270.

31 Moy ML, Israel E, Weiss ST, et al. Clinical predictors of health-related quality of life depend on asthma severity. Am J Respir Crit Care Med 2001; 163: 924-929.

32 Rosenzweig JRC, Edwards L, Lincourt W, et al. The relationship between health-related quality of life, lung function and daily symptoms in patients with persistent asthma. Respir Med 2004; 98: 1157-1165. 